\title{
Promotion of Early Childhood Development Using mHealth: Learnings From an Implementation Experience in Haryana
}

\author{
ViJay Kumar, Premananda Mohanty, Minakshi Sharma \\ From Survival for Women and Children Foundation, Sector 16, Panchkula, Haryana. \\ Correspondence to: Dr Vijay Kumar, Survival for Women and Children Foundation, Sector 16, Panchkula, Haryana. \\ 1940kumarv@gmail.com
}

\begin{abstract}
Pregnancy and the early years of life (0-3 years) are of crucial importance for a child's survival, health, growth and development. Improving care for young children is now considered fundamental to achieving the Sustainable Development Goals by 2030. With support from WHO and Intervida (an international non-governmental organization), implementation on care for early childhood development was carried out by Survival for Women and Children Foundation in 100 villages in Haryana, India. In addition to the implementation of evidence-based interventions, mHealth (phone message (SMS) and phone call) was used as a complementary strategy. The intention was to promote selfcare, increase coverage, and improve inter-sectoral collaboration. One message per day was developed (915 messages) and 1564630 SMS were sent to all beneficiaries and providers to facilitate interaction. Based on learnings, the consolidation of this approach into 46 core themes helped to refine interactions. Lot Quality Assurance Sampling was used for evaluation. SMS was received, read and practiced by the caregivers and the care providers in the intervention block, being substantially higher than in the control blocks. There was a remarkable improvement in under-nutrition and wasting; however, the reduction in stunting was modest in the intervention area as compared with two control blocks. This is attributed to implementation of all strategies in the project including the complementary approach of use of mHealth. The application of SMS and phone communication continues to have relevance, since people most in need are poor and require integrated package of services maximally during this crucial period for improving equity and coverage.
\end{abstract}

Keywords: Continuum of care, e-health, Integrated approach, Inter-sectoral collaboration, Self-care.

$\mathrm{P}$ regnancy and the early years of life (0-3 years) are of crucial importance for a child's survival, health, growth and development. It is a period of great opportunity, but also of vulnerability to negative influences. Many children do not reach their full develop-mental potential because of poverty, low maternal literacy, poor maternal mental health, lack of adequate nutrition, care, and opportunities to learn [1]. Improving care for young children is now considered fundamental to achieving the Sustainable Development Goals (SDGs) by 2030 [2]. Various studies and reports have recognized the importance of Early Childhood Development (ECD) to equity and greater success at school. In addition, as adults, they have greater employment opportunities and earnings besides better health, lower rates of crime, and lower levels of dependence on welfare programs [1]. There is an enormous return throughout life for small investment in the early years and substantial financial implications [3]. This makes a compelling case for mainstreaming healthcare for ECD in various national programs to achieve the goals of SDGs through inter-sectoral actions, with the health sector in the lead $[2,4]$.

\section{THE PROJECT}

In 2011, the World Health Organization (WHO) with support from Intervida supported the 'Care for Child Development' project between April, 2011 till December, 2014. Two sites, Survival for Women and Children Foundation (SWACH), Panchkula and Mahatma Gandhi Institute of Medical Sciences (MGIMS), Wardha were selected for implementation. A technical committee was constituted to represent the WHO, Ministry of Health and Family Welfare (MoHFW), National Institute of Public Cooperation and Child Development (NIPCCD), National Institute of Health and Family Welfare (NIHFW) for oversight. A training workshop was organized by WHO at MGIMS, Wardha for five days, with the participation of concerned stakeholders. The two sites implemented this project independently. This article refers to the learning experiences of SWACH. In addition to the implementation of evidence-based interventions, mHealth, in the form of short message services (SMS) and phone call, was used as a strategy, with the intention of promoting self-care, increasing coverage, and improving inter-sectoral collaboration. The aim of the project was to develop and implement an integrated package of ECD at grassroots level.

\section{PROJECT METHODOLOGY}

In Haryana, at the state level collaboration was established with the state National Health Mission (NHM), and at the district level collaboration with the Chief Medical Officer (CMO) and Integrated Child Development Services (ICDS) 
program officer. Bilaspur is a community develop-ment block in district Yamunanagar, Haryana. According to the census of 2011 [5], Bilaspur has a total population of 126791 people, out of which $115058(90.7 \%)$ live in the rural area. The schedule caste population constitutes $33.3 \%$ of the rural population. The overall literacy rate of rural Bilaspur is $64.3 \%$ (male literacy $70.1 \%$ and female literacy $57.8 \%$ ). The workforce in the block is mainly agricultural.

\section{Implementation}

The guiding principles in implementation include: (1) continuum of care across available delivery channels between 0-3 years; (2) an integrated approach with focus on ECD; (3) multi-sectoral coordination; (4) grassroot implementation; and (5) development of innovation for equitable coverage.

\section{Study Area and the Context}

The project was implemented in three phases in 100 villages of Bilaspur Community Development Block (101 893 population). Phase-I comprised of understanding the community, identifying the implementation strategy, and preparation for implementation. Implementation of the project in 10 villages was done initially and then expanded. In phase-III, the implementation was initially scaled up in 40 villages, which was then extended to 100 villages.

For the implementation of the project, health care providers included Accredited Social Health Activists (ASHAs) and their supervisors. ASHAs provided home visits and undertook health promotional work focusing on maternal and child health. Anganwadi workers (AWWs) and helpers under ICDS provided nutrition and other services in the villages. Community leaders from Panchayati Raj Institutions (PRI) were involved to facilitate implementation in the villages. Voluntary mothers' club was an innovation of the SWACH project, with 96 mothers' clubs being formed across 100 villages. These clubs were voluntary groups of 10-12 women of the village who were either pregnant or had children less than 3 years of age. Each club had a leader who was selected by the members. The objective to form these groups was to increase the outreach of beneficiaries. During the implementation of the project, keeping all the above workforce and volunteers, engaged with coordination was a challenge that needed to be addressed.

Within the umbrella of this project, a complementary strategy of using mobile phone calls was used with the following objectives: $i$ ) to develop, transmit, use and assess SMS and phone communication to complement the implementation; $i$ ) to explore feasibility of integration and inter-sectoral collaboration by using SMS and phone calls; and iii) to expand the coverage by strengthening of the services and the home visits based on learning from the community.

\section{Message of the Day - Development, Transmission and Utilization}

SMS was a feasible option available at that time to cover an important agenda of the ECD, which was to provide correct information to the target audience. It was decided to send messages by phone to the families and providers to empower families in self-care, strengthen ongoing capacity development of health care providers in ECD, and bring about better coordination among the numerous providers who were involved in various activities (home visits, anganwadi meetings, voluntary mother club meetings and other outreach sessions).

The project staff along with a senior supervisor at SWACH developed the messages from various material i.e., Facts for Life [6], WHO- United Nations Children's Fund (UNICEF) Care for Child Development package [7], Integrated Management of Neonatal and Childhood Illness (IMNCI) package, developed by India, WHO Healthy Child Development package [8] and Mother Child Protection (MCP) card [9]. The messages developed were evidence-based and in conformity to the state and national policy. The messages related to one or more of the following five components of the integrated package: play and communication; feeding; prevention of injuries and diseases; timely recognition and treatment of illnesses; and, pregnancy. The messages were refined to ensure simplicity of the language, correctness and appropriateness of information. The messages were in English script using a mixture of Hindi and English words since, at that time, writing in Hindi was a technical barrier. Local terms were also incorporated. The text was limited to 160 characters.

The target audience included all families of caregivers with children below 3 years of age, pregnant women, frontline care providers (Health and ICDS), doctors, program managers, other stakeholders and SWACH project staff. Consent was obtained for participation. Ninety per cent of the targeted population had mobile phones. Its ownership was spread evenly across different socioeconomic strata.

Based on the registration, line listing of the households was done, and their phone numbers were entered along with other details. The project coordinator transmitted the message via phone to the staff daily. Then the concerned staff members sent the message of the day to the target audience assigned to them. Strategically, on a few days, the messages were coordinated with state, national and international programs like breastfeeding week, diarrhea fortnight, immunization days, polio campaign, mother's day etc. to boost the efforts made by the state and national program. 
During the scale-up phase, the number of beneficiaries increased. It was not feasible to send a message of the day to a large number of families and providers through personal phones. Therefore, during phase II and III, we switched to the transmission of SMS through the internet daily, messages were not received by those families who had opted for a Do Not Disturb (DND) facility on their mobile. Messages to health workers, AWWs and mothers' clubs' leaders were sent to their mobile numbers by SWACH staff to overcome this problem. To incorporate integration of messages into angan-wadi workers and home visits by providers, group discussion and meetings were promoted and held daily. Apart from this, voluntary mothers' clubs' meetings were held once in a week in which the message of the day was read out and was open for discussion.

\section{Theme of the Day}

On the basis of continuous quality assessment and feedback received from the providers, it was felt that a daily message of 160 characters was not sufficient to trigger meaningful discussion. Therefore, it was decided to equip the health care providers (ASHA and AWW) and leaders of the voluntary mothers' club with sufficient information in the form of two page note on each theme, so that the discussion during the group meetings or home visit becomes more effective and focused. This two-page note called 'theme of the day' was developed in simple local language after consulting national and international literature on best practices. Priority themes were identified from all the components of the integrated package. A total of 46 themes were shortlisted and the content was developed addressing their importance. The theme was kept simple, easily understood for translation into practice. A consensus was reached to send a message of 320 characters on each theme for a better coordination in the field. The package, in the form of booklet, was distributed to all care providers by SWACH staff. They were then implemented by the SWACH staff, health care providers and other stakeholders. To implement thematic discussion, a promotional message was sent one day in advance, so that the provider got familiar with it and prepared the theme before conducting the group session or home visit. The care providers used the message as a talking point during discussions when they conducted home visits or group sessions.

Monitoring of meetings and home visits was done by the SWACH staff. Three teams of two supervisors each evaluated quality of group meetings and home visits twice-a-week.

A household survey was conducted to register targeted families and, the following parameters relevant to the study were documented: $a$ ) Received message; $b$ ) Message read; c) Considered for practice; $d$ ) Phone call to SWACH for consultation.

\section{Assessment Using Lot Quality Assurance Sampling (LQAS)}

This method was used for assessing the quality of the project. Four age bands were considered - 0-5 month, 6-11 month, 12-23 month, and 24-35 month. The last LQAS survey was considered equivalent to the end line survey for the project. A sample size of 95 was required, 19 in each band for 5 different supervisory areas [10]. In this study, sample of 20 was taken for each supervisory area for ease of calculation, totaling to 100 . Simple random sampling was utilized. Three schedules were utilized as per recommendation. These included several parameters including assessment of malnutrition (under-nutrition, wasting including mid upper arm circumference and stunting).

Since, baseline information collected through household survey was incomplete for evaluation of messages, it was included in LQAS. Two control community development blocks (Mullana and Khizrabad) with similar health, ICDS infrastructure and interventions (like MCP card, socio-economic demographic characteristics) were included to minimize biases related to socioeconomic characteristics and health care delivery system.

\section{PROJECTRESULTS}

In phase-I and II, 501 families and 112 providers were included. This increased to 1291 families and 209 providers initially in phase-III, and expanded to 3767 families and 392 providers later in the phase.

Over a period of 34 months, a total of 915 messages were developed till the end of the project and 15,64,630 SMS were sent to the beneficiaries and providers in the study area. There was a progressive increase in the number, as the project was scaled up from 10 to 100 villages. Web Fig. 1 depicts the distribution of message content according to the specific aspect of healthcare covered.

SMS was received, read and practiced by the caregivers and the care providers, whereas in both the control areas, receipt of the message was very low $(5 \%$ and $2 \%$ ). These messages were likely sent by other agencies or shared by the families to their relatives in the neighbouring blocks. There was improved coverage in home visit by ASHA, and also visit of caregivers to the anganwadi center in the intervention area compared to the two control areas. The need for ASHA home visit, anganwadi meeting and mothers' club was strongly felt in all the groups by the beneficiaries. The proportion of families and providers who received the messages and utilized them during group meetings and at home are summarized in Table I. 
Table I Results Related to Short Message Service During the Evaluation

\begin{tabular}{|c|c|c|c|c|c|}
\hline \multirow[t]{2}{*}{ Category } & \multirow[t]{2}{*}{ HH survey } & \multirow[t]{2}{*}{$L Q A S-1$} & \multirow[b]{2}{*}{ Intervention area } & \multicolumn{2}{|l|}{$L Q A S$-final } \\
\hline & & & & Control area 1 & Control area 2 \\
\hline \multicolumn{6}{|l|}{ Care givers } \\
\hline Received message & 1.2 & 72.8 & 68.0 & 5.0 & 2.0 \\
\hline Message read & 10.0 & 94.5 & 60.0 & 5.0 & 2.0 \\
\hline Considered for practice & 5.0 & 50.0 & 31.0 & 4.0 & 1.0 \\
\hline Phone call to SWACH for consultation & 15.0 & 55.3 & 62.0 & 7.0 & 4.0 \\
\hline \multicolumn{6}{|l|}{$A S H A$} \\
\hline Visited home & NA & 80.0 & 82.0 & 65.0 & 62.0 \\
\hline Message read & NA & 42.8 & 62.0 & 23.0 & 25.0 \\
\hline Discussion around message & NA & 42.0 & 56.0 & 23.0 & 17.0 \\
\hline Is an ASHA visit required & NA & 94.2 & 100.0 & 98.0 & 94.0 \\
\hline \multicolumn{6}{|l|}{ Anganwadi center } \\
\hline Caregiver visited AWC & NA & 17.2 & 26.0 & 6.0 & 8.0 \\
\hline Message read by AWW & NA & 39.2 & 22.0 & 0 & 0 \\
\hline Activities on play \& communication & NA & 34.2 & 22.0 & 3.0 & 4.0 \\
\hline Practice by caregiver & NA & 40.0 & 24.0 & 3.0 & 5.0 \\
\hline Is Anganwadi meeting useful & NA & 87.0 & 99.0 & 100.0 & 100.0 \\
\hline \multicolumn{6}{|l|}{ Mothers' club } \\
\hline Is mothers' club required & NA & 91.0 & 98.0 & 99.0 & 95.0 \\
\hline Membership & NA & 43.0 & 44.0 & 0 & 0 \\
\hline Meeting attended & NA & 43.0 & 44.0 & 0 & 0 \\
\hline Message read by mothers' club leader & NA & 39.5 & 41.0 & 0 & 0 \\
\hline Activity by MC leader around ECD & NA & 39.8 & 41.0 & 0 & 0 \\
\hline Practice by parents & NA & 41.3 & 38.0 & 0 & 0 \\
\hline
\end{tabular}

Data presented in percentages. LQAS: Lot quality assurance sampling; LQAS-1 during December, 2012 and LQAS - final during December, 2014. ASHA: Accredited social health activists; AWC: Anganwadi centre; AWW: anganwadi worker; ECD: early childhood development; HH: house hold; MC: mothers' club; SWACH: survival for women and children foundation.

There was a remarkable improvement in undernutrition and wasting; however, the reduction in stunting was modest in the intervention area Bilaspur, as compared with control blocks Khizrabad and Mullana (Web Fig. 2).

\section{Learning from the Project}

The use of digital technology and its application in health promotion and health care has been evolving. The aim of the project was to develop and implement an integrated package of ECD using a continuum of care approach at grassroots level. Based on learning from the community to improve a child's survival, healthy growth and development as well as care giver's health. The objectives of complementary strategy of mHealth were: to use SMS and phone communication to explore inter-sectoral collabo-ration; and to strengthen services and home visits.

The messages were developed following a review of national and international literature on best practices [7-9]. They were adapted according to culture and were written in English script (suitable option available at that time) and consolidated into less than 160 characters as per permitted limits. Balanced approach was used in transmission with emphasis on ECD and nutrition. The messages developed were transmitted through internet to all beneficiaries and care providers at all levels on a daily basis. The limitation of 100 messages per day per mobile phone was overcome by sending bulk messages through internet. The problem of DND was partially overcome by sending messages by the SWACH staff to all the care providers.

The transmitted messages were targeted to the beneficiaries to trigger discussion around the message during home visits by ASHAs and become a part of group discussions in anganwadi meetings, village health, nutrition day meetings and voluntary mothers' clubs meetings.

The feedback from quality assessment and observations helped to address the inadequacies related to SMS. To overcome this problem and to strengthen the discussion and interaction, theme-based package was developed and distributed to all the health care providers for use during home visits by ASHAs, Anganwadi meetings and voluntary mothers' club's meetings. Evaluation showed remarkable improvement in weight for age, wasting and modest 
improvement in stunting in the intervention block as compared to two control blocks. These improvements can be attributed to utilization of evidence-based interventions (vaccination, breastfeeding, regular weighing, use of MCP card, timely treatment of illnesses, etc.). The complementary strategy (daily SMS, telephone calls and thematic discussions) for larger engagement of the beneficiaries on an ongoing basis combined with integration and intersectoral collaboration, most likely helped upgrade self-care and timely care-seeking.

At that time possession of smart phones was uncommon, although feature phones were available with vast majority of families and providers. The internet connectivity was erratic and there was no widespread use of mobile applications. The script of the message was in English, many beneficiaries were unable to read it. Family members and providers helped the beneficiaries to overcome this problem.

While developing the message, limiting the text to 160 characters was challenging. The transmission of messages that were age-specific was not relevant with the families of children in a different age band. This problem could not be overcome, since only one message was transmitted per day.

Throughout the project, to learn feasibility of implementation, evidence-based practices at grassroots level, advantage was taken of the available packages and information on best practices fromUNICEF, WHO and Ministry of Health [1,7-9].

Various studies including India [11-14] on the use of mobile technology (SMS, phone calls, or other mobile applications) for health assistance indicate that the findings and learnings of the present article are valid and in line with the literature. However, a systematic review [15] indicates that impact and potential use of mHealth has not been fully utilized by the care givers and the care seekers. The application of SMS and phone communication continues to have relevance even now, since people in need, do not have smart phones, but are in maximal need of integrated package of services during this crucial period. This is one of the strategies to be considered in efforts to obtain equity and improved coverage.

In the present day COVID-19 crisis, where in-person contact is avoided and social distancing is promoted, utilizing the technology for health care assistance in terms of SMS and phone calls can be valuable for promoting and monitoring the health of the mother and the baby.

Acknowledgements: Dr. Richa Mukhra assisted in reviewing, revising and finalizing the manuscript. Mr. Deepak Kumar provided coordination in paper writing through digital support.

Funding: WHO and Intervida; Competing interests: None stated.

\section{REFERENCES}

1. Grantham-McGregor S, International Child Development Committee. Early child development in developing countries. Lancet. 2007;369:824.

2. GBD 2015 SDG Collaborators. Measuring the health-related sustainable development goals in 188 countries: A baseline analysis from the global burden of disease study 2015. Lancet. 2016;388: 1813-50.

3. McGrath M. Advancing early childhood development: from science to scale. An executive summary for the lancet's series. Lancet. 2016.

4. Britto PR, Lye SJ, Proulx K, et al. Nurturing care: promoting early childhood development. Lancet. 2017;389:91-102.

5. Office of the Registrar General and Census Commissioner, Ministry of Home Affairs, Government of India. Census of India 2011. Accessed May 1, 2021. Available from: http://censusindia.gov.in/ 2011-Common/CensusData2011.html

6. The Mother and Child Health and Education Trust. Facts for life. Accessed May 1, 2021. Available from: https://factsforlife.org/

7. World Health Organization. Care for child development: improving the care of young children. WHO; 2012.

8. World Health Organization. Care for child's healthy growth and development. World Health Organization; 2015.

9. Ministry of Health and Family Welfare, Ministry of Women and Child Development. Guidebook for Mother Child Protection Card 2018. Accessed May 1, 2021. Available from: https://nhm.gov.in/ New_Updates_2018/NHMComponents/Immunization/Guildelines for_immunization/MCP_Guide_Book.pdf

10. Valadez JJ, Weiss W, Leburg C, et al. Assessing Community Health Programs: Using LQAS for Baseline Survey and Regular Monitoring, 2nd ed. Teaching Aids At Low Costs (TALC); 2007.

11. Aggarwal R, Sharma AK, Guleria K. Antenatal care during the pandemic in India: the problem and the solutions. Int J Pregn and Chi Birth. 2021;7:15-17.

12. Ceballos F, Hernandez MA, Olivet F, et al. Assessing the use of cell phones to monitor health and nutrition interventions: Evidence from rural Guatemala. PLoS One. 2020;15:e0240526.

13. Tamrat T, Kachnowski S. Special delivery: an analysis of mHealth in maternal and newborn health programs and their outcomes around the world. Matern Child Health J. 2012;16:1092-101.

14. Poorman E, Gazmararian J, Parker RM, et al. Use of text messaging for maternal and infant health: A systematic review of the literature. Matern Child Health J. 2015;19:969-89.

15. Haddad SM, Souza RT, Cecatti JG. Mobile technology in health (mHealth) and antenatal care-Searching for apps and available solutions: A systematic review. Int J Med Inform. 2019;127:1-8. 